\title{
On Square Divisor Cordial Graphs
}

\author{
S. K. Vaidya ${ }^{1 *}$ and N. H. Shah ${ }^{2}$ \\ ${ }^{1}$ Department of Mathematics, Saurashtra University, Rajkot-360005, Gujarat (INDIA) \\ ${ }^{2}$ Government Polytechnic, Rajkot-360003, Gujarat (INDIA)
}

Received 14 September 2013, accepted in revised form 22 June 2014

\begin{abstract}
The square divisor cordial labeling is a variant of cordial labeling and divisor cordial labeling. Here we prove that the graphs like flower $F l_{n}$, bistar $B_{n, n}$, restricted square graph of $B_{n, n}$, shadow graph of $B_{n, n}$ as well as splitting graphs of star $K_{1, n}$ and bistar $B_{n, n}$ are square divisor cordial graphs. Moreover we show that the degree splitting graphs of $B_{n, n}$ and $P_{n}$ admit square divisor cordial labeling.

Keywords: Square divisor cordial labeling, Divisor cordial labeling.

() 2014 JSR Publications. ISSN: 2070-0237 (Print); 2070-0245 (Online). All rights reserved.

doi: http://dx.doi.org/10.3329/jsr.v6i3.16412 J. Sci. Res. 6 (3), 445-455 (2014)
\end{abstract}

\section{Introduction}

We begin with simple, finite, connected and undirected graph $G=(V(G), E(G))$ with $p$ vertices and $q$ edges. For standard terminology and notations related to graph theory we refer to Gross and Yellen [1] while for any concept of number theory we refer to Burton [2]. We will provide brief summary of definitions and other information which are prerequisites for the present investigations.

Definition 1.1 If the vertices are assigned values subject to certain condition(s) then it is known as graph labeling.

The graph labeling is described as a frontier between number theory and structure of graphs by Beineke and Hegde [3]. There are enormous applications of graph labeling in various fields including computer science and communication networks. Yegnanaryanan and Vaidhyanathan [4] described applications of edge balanced graph labeling, edge

\footnotetext{
*Corresponding author: samirkvaidya@yahoo.co.in
} 
magic labeling and $(1,1)$ edge magic graph. For a dynamic survey of various graph labeling problems along with an extensive bibliography we refer to Gallian [5].

Definition 1.2 A mapping $f: V(G) \rightarrow\{0,1\}$ is called binary vertex labeling of $G$ and $f(v)$ is called the label of the vertex $v$ of $G$ under $f$.

Notation 1.3 If for an edge $e=u v$, the induced edge labeling $f^{*}: E(G) \rightarrow\{0,1\}$ is given by $f^{*}(e)=|f(u)-f(v)|$. Then

$v_{f}(i)=$ number of vertices of $G$ having label $i$ under $f$

$e_{f}(i)=$ number of vertices of $G$ having label $i$ under $f *$

Definition 1.4 A binary vertex labeling $f$ of a graph $G$ is called a cordial labeling if $\left|v_{f}(0)-v_{f}(1)\right| \leq 1$ and $\left|e_{f}(0)-e_{f}(1)\right| \leq 1$. A graph $G$ is cordial if it admits cordial labeling.

The concept of cordial labeling was introduced by Cahit [6]. This concept has been explored by many researchers and various labeling schemes are also introduced with minor variations in cordial theme. Product cordial labeling, total product cordial labeling and prime cordial labeling and divisor cordial labeling are among mention a few. The present work is focused on square divisor cordial labeling.

Definition 1.5 A prime cordial labeling of a graph $G$ with vertex set $V(G)$ is a bijection $f: V(G) \rightarrow\{1,2,3, \ldots,|V(G)|\}$ and the induced function $f^{*}: E(G) \rightarrow\{0,1\}$ is defined by

$$
\begin{aligned}
f *(e=u v) & =1, \text { if } \operatorname{gcd}(f(u), f(v))=1 ; \\
& =0, \text { otherwise }
\end{aligned}
$$

which satisfies the condition $\left|e_{f}(0)-e_{f}(1)\right| \leq 1$. A graph which admits prime cordial labeling is called a prime cordial graph.

The concept of prime cordial labeling was introduced by Sundaram et al. [7] and in the same paper they have investigated several results on prime cordial labeling. Vaidya and Vihol $[8,9]$ as well as Vaidya and Shah $[10,11]$ have proved many results on prime cordial labeling.

Varatharajan et al. [12] have introduced a new concept called divisor cordial labeling by combining the divisibility of numbers and the concept of Cordial labeling.

Definition 1.6 Let $G=(V(G), E(G))$ be a simple graph and $f: \rightarrow\{1,2, \ldots|V(G)|\}$ be a bijection. For each edge $u v$, assign the label 1 if $f(u) \mid f(v)$ or $f(v) \mid f(u)$ and the label 0 otherwise. $f$ is called a divisor cordial labeling if $\left|e_{f}(0)-e_{f}(1)\right| \leq 1$. A graph with a divisor cordial labeling is called a divisor cordial graph.

In the same paper [12] they have proved that path, cycle, wheel, star, $K_{2, n}$ and $K_{3, n}$ are divisor cordial graphs while $K_{n}$ is not divisor cordial for $n \geq 7$. Same authors in [13] have discussed divisor cordial labeling of full binary tree as well as some star related graphs. Vaidya and Shah [14] have proved that some star and bistar related graphs are 
divisor cordial graph. Same authors [15] have shown that helm, flower and gear graphs admit divisor cordial labeling. Moreover the graphs obtained from switching of a vertex in various graphs are proved to be divisor cordial.

Motivated by the concept of divisor cordial labeling, Murugesan et al. [16] have introduced the concept of square divisor cordial labeling and many graphs are proved to be square divisor cordial graphs.

Definition 1.7 Let $G=(V(G), E(G))$ be a simple graph and $f: \rightarrow\{1,2, \ldots|V(G)|\}$ be a bijection. For each edge $u v$, assign the label 1 if $f(u)^{2} \mid f(v)$ or $f(v)^{2} \mid f(u)$ and the label 0 otherwise. $f$ is called a square divisor cordial labeling if $\left|e_{f}(0)-e_{f}(1)\right| \leq 1$. A graph with a square divisor cordial labeling is called a square divisor cordial graph.

Definition 1.8 The flower $F l_{n}$ is the graph obtained from a helm $H_{n}$ by joining each pendant vertex to the apex of the helm. It contains three types of vertices: an apex of degree $2 n, n$ vertices of degree 4 and $n$ vertices of degree 2 .

Definition 1.9 Bistar $B_{n, n}$ is the graph obtained by joining the center (apex) vertices of two copies of $K_{1, n}$ by an edge.

Definition 1.10 For a simple connected graph $G$ the square of graph $G$ is denoted by $G^{2}$ and defined as the graph with the same vertex set as of $G$ and two vertices are adjacent in $G^{2}$ if they are at a distance 1 or 2 apart in $G$.

Definition 1.11 The shadow graph $D_{2}(G)$ of a connected graph $G$ is constructed by taking two copies of $G$ say $G^{\prime}$ and $G^{\prime \prime}$. Join each vertex $u^{\prime}$ in $G^{\prime}$ to the neighbours of the corresponding vertex $v^{\prime}$ in $G^{\prime \prime}$.

Definition 1.12 For a graph $G$ the splitting graph $S^{\prime}(G)$ of a graph $G$ is obtained by adding a new vertex $v^{\prime}$ corresponding to each vertex $v$ of $G$ such that $N(v)=N\left(v^{\prime}\right)$.

Definition 1.13 [17] Let $G=(V(G), E(G))$ be a graph with $V=S_{1} \cup S_{2} \cup S_{3} \cup \ldots S_{t} \cup T$ where each $S_{i}$ is a set of vertices having at least two vertices of the same degree and $T=V-\left(\cup_{i=1}^{t} S_{i}\right)$. The degree splitting graph of $\mathrm{G}$ denoted by $D S(G)$ is obtained from $G$ by adding vertices $w_{1}, w_{2}, w_{3}, \ldots, w_{t}$ and joining to each vertex of $S_{i}$ for $1 \leq i \leq t$. 


\section{Main Results}

Theorem 2.1 : Flower graph $F l_{n}$ is a square divisor cordial graph for each $n$.

Proof: Let $v$ be the apex, $v_{1}, v_{2}, \ldots, v_{n}$ be the vertices of degree 4 and $u_{1}, u_{2}, \ldots, u_{n}$ be the vertices of degree 2 of $F l_{n}$. Then $\left|V\left(F l_{n}\right)\right|=2 n+1$ and $\left|E\left(F l_{n}\right)\right|=4 n$. We define vertex labeling $f: V(G) \rightarrow\{1,2,3, \ldots, 2 n+1\}$ as follows.

$$
\begin{aligned}
& f v=1, \\
& f\left(v_{1}\right)=2, \\
& f\left(u_{1}\right)=3, \\
& f\left(v_{1+i}\right)=5+2(i-1) ; \quad 1 \leq i \leq n-1 \\
& f\left(u_{1+i}\right)=4+2(i-1) ; \quad 1 \leq i \leq n-1
\end{aligned}
$$

In view of the above labeling pattern we have,

$e_{f}(0)=2 n=e_{f}(1)$. Thus, $\left|e_{f}(0)-e_{f}(1)\right| \leq 1$.

Hence $F l_{n}$ is a square divisor cordial graph for each $n$.

Illustration 2.2 Square divisor cordial labeling of the graph $F l_{11}$ is shown in Fig. 1.

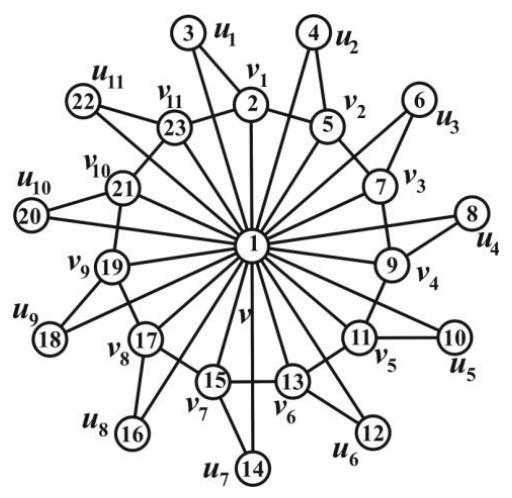

Fig. 1.

Theorem 2.3: $B_{n, n}$ is a square divisor cordial graph.

Proof : Consider $B_{n, n}$ with vertex set $\left\{u, v, u_{i}, v_{i}, 1 \leq i \leq n\right\}$ where $u_{i}, v_{i}$ are pendant vertices. If $G=B_{n, n}$ then $|V(G)|=2 n+2$ and $|E(G)|=2 n+1$. We define vertex labeling $f: V(G) \rightarrow\{1,2, \ldots, 2 n+2\}$ as follows. 


$$
\begin{array}{ll}
f(u)=1, & \\
f(v)=2 n+1, & \\
f\left(u_{i}\right)=1+i ; & 1 \leq i \leq n \\
f\left(v_{i}\right)=n+1+i ; & 1 \leq i \leq n-1 \\
f\left(v_{n}\right)=2 n+2 ; &
\end{array}
$$

In view of the above labeling pattern we have, $e_{f}(0)=n+1, e_{f}(1)=n$. Thus, $\left|e_{f}(0)-e_{f}(1)\right| \leq 1$.

Hence $B_{n, n}$ is a square divisor cordial graph.

Illustration 2.4 : Square divisor cordial labeling of the graph $B_{8,8}$ is shown in Fig. 2.

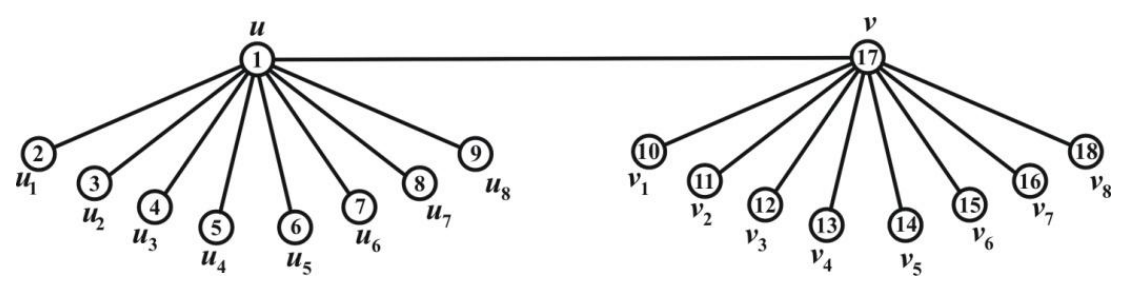

Fig. 2

Theorem 2.5 : Restricted $B_{n, n}^{2}$ is a square divisor cordial graph.

Proof : Consider $B_{n, n}$ with vertex set $\left\{u, v, u_{i}, v_{i}, 1 \leq i \leq n\right\}$ where $u_{i}, v_{i}$ are pendant vertices. Let $G$ be the restricted $B_{n, n}^{2}$ graph with $V(G)=V\left(B_{n, n}\right)$ and $E(G)=E\left(B_{n, n}\right) \cup\left\{v u_{i}, u v_{i} / 1 \leq i \leq n\right\}$ then $|V(G)|=2 n+2$ and $E(G)=4 n+1$.

We define vertex labeling $f: V(G) \rightarrow\{1,2, \ldots, 2 n+2\}$ as follows.

Let $p_{1}$ be the highest prime number $<2 n+2$.

$$
\begin{aligned}
& f(u)=1, \\
& f(v)=p_{1}, \\
& f\left(u_{1}\right)=2, \\
& f\left(v_{i}\right)=4+2(i-1) ; \quad 1 \leq i \leq n
\end{aligned}
$$

For the vertices $u_{2}, u_{3}, \ldots, u_{n}$ we assign distinct odd numbers (except $p_{1}$ ). In view of the above defined labeling pattern we have, $e_{f}(0)=2 n, e_{f}(1)=2 n+1$. Thus, $\left|e_{f}(0)-e_{f}(1)\right| \leq 1$.

Hence $B_{n, n}^{2}$ is a square divisor cordial graph.

Illustration 2.6: Square divisor cordial labeling of restricted $B_{7,7}^{2}$ is shown in Fig. 3 . 


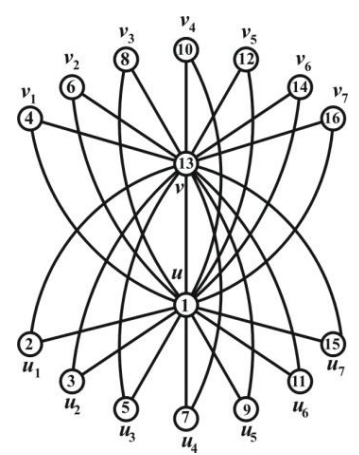

Fig. 3

Theorem 2.7: $D_{2}\left(B_{n, n}\right)$ is a square divisor cordial graph.

Proof: Consider two copies of $B_{n, n}$. Let $\left\{u, v, u_{i}, v_{i}, 1 \leq i \leq n\right\}$ and $\left\{u^{\prime}, v^{\prime}, u_{i}^{\prime}, v_{i}^{\prime}, 1 \leq i \leq n\right\}$ be the corresponding vertex sets of each copy of $B_{n, n}$. Let $G$ be the graph $D_{2}\left(B_{n, n}\right)$ then $|V(G)|=4(n+1)$ and $|E(G)|=4(2 n+1)$. We define vertex labeling $f: V(G) \rightarrow\{1,2, \ldots, 4(n+1)\}$ as follows.

Let $p_{1}$ be the highest prime number, $p_{2}$ be the second highest prime number and $p_{3}$ be the third highest prime number such that $p_{3}<p_{2}<p_{1}<4(n+1)$.

$$
\begin{array}{ll}
f(u)=p_{1}, & f\left(u^{\prime}\right)=1, \\
f(v)=p_{2}, & f\left(v^{\prime}\right)=p_{3}, \\
f\left(u_{i}\right)=2+2(i-1) ; & 1 \leq i \leq n \\
f u_{i}^{\prime}=f\left(u_{n}\right)+2 i ; & 1 \leq i \leq n \\
f\left(v_{1}\right)=4(n+1), & f\left(v_{2}\right)=4 n,
\end{array}
$$

For the vertices $v_{3}, v_{4}, \ldots, v_{n}$ and $v_{1}^{\prime}, v_{2}^{\prime}, \ldots, v_{n}^{\prime}$ we assign distinct odd numbers (except $p_{1}, p_{2}$ and $p_{3}$ ).

In view of the above defined labeling pattern we have, $e_{f}(0)=4 n+2=e_{f}(1)$. Thus, $\left|e_{f}(0)-e_{f}(1)\right| \leq 1$.

Hence $D_{2}\left(B_{n, n}\right)$ is a square divisor cordial graph.

Illustration 2.8: Square divisor cordial labeling of the graph $D_{2}\left(B_{5,5}\right)$ is shown in Fig. 4 . 


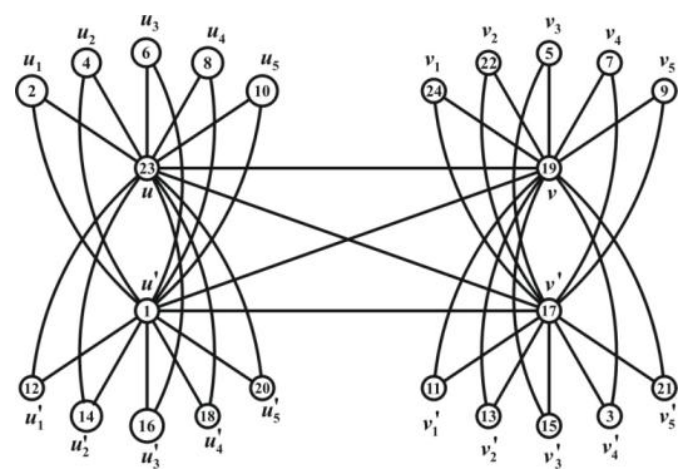

Fig. 4

Theorem 2.9: $S^{\prime}\left(K_{1, n}\right)$ is a square divisor cordial graph.

Proof : Let $v_{1}, v_{2}, v_{3}, \ldots, v_{n}$ be the pendant vertices and $v$ be the apex vertex of $K_{1, n}$ and $u, u_{1}, u_{2}, u_{3}, \ldots, u_{n}$ are added vertices corresponding to $v, v_{1}, v_{2}, v_{3}, \ldots, v_{n}$ to obtain $S^{\prime}\left(K_{1, n}\right)$.

Let $G$ be the graph $S^{\prime}\left(K_{1, n}\right)$ then $|V(G)|=2 n+2$ and $|E(G)|=3 n$. We define vertex labeling $f: V(G) \rightarrow\{1,2, \ldots, 2 n+2\}$ as follows.

$$
\begin{array}{ll}
f(v)=2, & \\
f(u)=1, & \\
f\left(v_{1+i}\right)=4+2 i ; & 0 \leq i<n \\
f\left(u_{1+i}\right)=3+2 i ; & 0 \leq i<n
\end{array}
$$

In view of the above defined labeling pattern we have,

$e_{f}(1)=n+\left\lfloor\frac{2 n+2}{4}\right\rfloor, e_{f}(0)=3 n-e_{f}(1)$.

Thus, $\left|e_{f}(0)-e_{f}(1)\right| \leq 1$.

Hence $S^{\prime}\left(K_{1, n}\right)$ is a square divisor cordial graph.

Illustration 2.10: Square divisor cordial labeling of the graph $S^{\prime}\left(K_{1,11}\right)$ is shown in Fig. 5 .

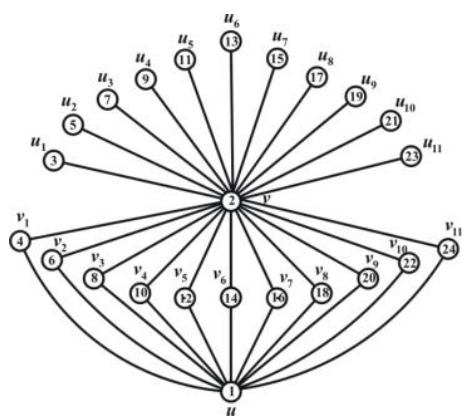

Fig. 5 
Theorem 2.11: $S^{\prime}\left(B_{n, n}\right)$ is a square divisor cordial graph.

Proof: Consider $B_{n, n}$ with vertex set $\left\{u, v, u_{i}, v_{i}, 1 \leq i \leq n\right\}$ where $u_{i}, v_{i}$ are pendant vertices. In order to obtain $S^{\prime}\left(B_{n, n}\right)$, add $u^{\prime}, v^{\prime}, u_{i}^{\prime}, v_{i}^{\prime}$ vertices corresponding to $u, v, u_{i}, v_{i}$ where, $1 \leq i \leq n$. If $G=S^{\prime}\left(B_{n, n}\right)$ then $|V(G)|=4(n+1)$ and $|E(G)|=6 n+3$. We define vertex labeling $f: V(G) \rightarrow\{1,2, \ldots, 4(n+1)\}$ as follows.

Let $p_{1}$ be the highest prime number and $p_{2}$ be the second highest prime number such that $\mathrm{p}_{2}<\mathrm{p}_{1}<4 \mathrm{n}+1$.

$$
\begin{aligned}
& f(u)=p_{2}, \\
& f\left(u^{\prime}\right)=2, \\
& f(v)=1, \\
& f\left(v^{\prime}\right)=p_{1}, \\
& f\left(u_{i}\right)=4+4(i-1) ; \quad 1 \leq i \leq n \\
& f u_{i}^{\prime}=6+4(i-1) ; \quad 1 \leq i \leq n \\
& f\left(v_{1}\right)=4 n+4,
\end{aligned}
$$

For the vertices $v_{2}, v_{3}, \ldots, v_{n}$ and $v_{1}^{\prime}, v_{2}^{\prime}, \ldots, v_{n}^{\prime}$ we assign distinct odd numbers (except $p_{1}$ and $\left.p_{2}\right)$.

In view of the above labeling pattern we have,

$e_{f}(0)=3 n+1, e_{f}(1)=3 n+2$.

Thus, $\left|e_{f}(0)-e_{f}(1)\right| \leq 1$.

Hence $S^{\prime}\left(B_{n, n}\right)$ is a square divisor cordial graph.

Illustration 2.12: Square divisor cordial labeling of the graph $S^{\prime}\left(B_{6,6}\right)$ is shown in Fig. 6.

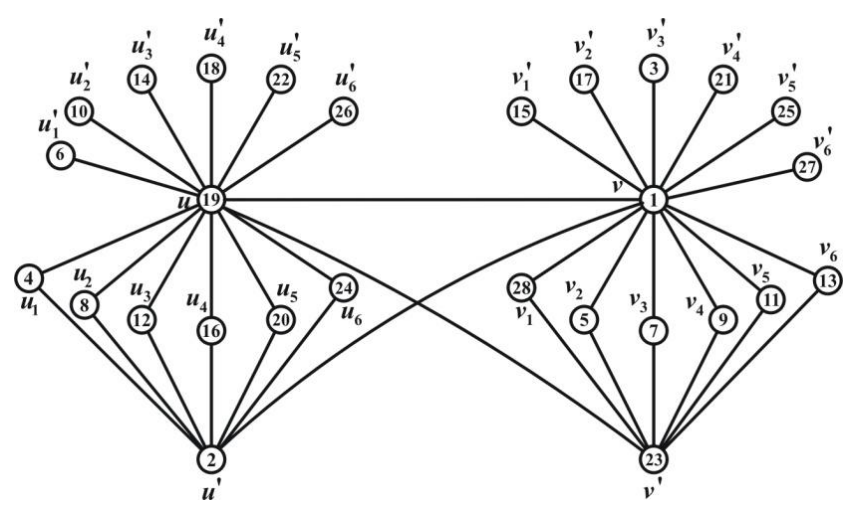

Fig. 6 
Theorem 2.13: $\operatorname{DS}\left(B_{n, n}\right)$ is a square divisor cordial graph.

Proof: Consider $B_{n, n}$ with $V\left(B_{n, n}\right)=\left\{u, v, u_{i}, v_{i}: 1 \leq i \leq n\right\}$, where $u_{i}, v_{i}$ are pendant vertices. Here $V\left(B_{n, n}\right)=V_{1} \cup V_{2}$, where $V_{1}=\left\{u_{i}, v_{i}: 1 \leq i \leq n\right\}$ and $V_{2}=\{u, v\}$. Now in order to obtain $D S\left(B_{n, n}\right)$ from $G$, we add $w_{1}, w_{2}$ corresponding to $V_{1}, V_{2}$. Then $\mid V\left(D S\left(B_{n, n}\right) \mid=2 n+4\right.$ and $E\left(D S\left(B_{n, n}\right)\right)=\left\{u v, u w_{2}, v w_{2}\right\} \cup\left\{u u_{i}, v v_{i}, w_{1} u_{i}, w_{1} v_{i}: 1 \leq i \leq n\right\}$ so $\mid E\left(D S\left(B_{n, n}\right) \mid=4 n+3\right.$. We define vertex labeling $f: V\left(D S\left(B_{n, n}\right)\right) \rightarrow\{1,2, \ldots, 2 n+4\}$ as follows.

$$
\begin{aligned}
& f(u)=4, \\
& f(v)=2 n+3, \\
& f\left(w_{1}\right)=1, \\
& f\left(w_{2}\right)=2, \\
& f\left(u_{i}\right)=3+2(i-1) ; \quad 1 \leq i \leq n \\
& f\left(v_{i}\right)=6+2(i-1) ; \quad 1 \leq i \leq n
\end{aligned}
$$

In view of the above defined labeling pattern we have,

$$
e_{f}(0)=2 n+2, e_{f}(1)=2 n+1 \text {. }
$$

Thus, $\left|e_{f}(0)-e_{f}(1)\right| \leq 1$.

Hence $\operatorname{DS}\left(B_{n, n}\right)$ is a square divisor cordial graph.

Illustration 2.14: Square divisor cordial labeling of the graph $D S\left(B_{5,5}\right)$ is shown in Fig. 7.

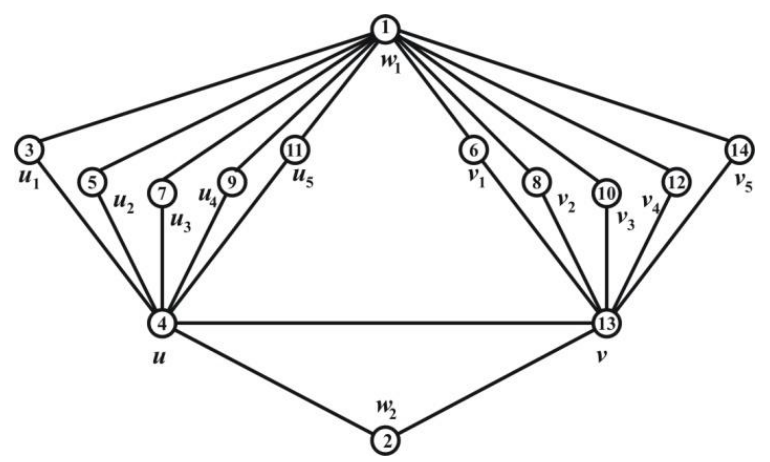

Fig. 7

Theorem 2.15: $D S\left(P_{n}\right)$ is a square divisor cordial graph. 
Proof : Consider $P_{n}$ with $V\left(P_{n}\right)=\left\{v_{i}: 1 \leq i \leq n\right\}$. Here $V\left(P_{n}\right)=V_{1} \cup V_{2}$, where $V_{1}=\left\{v_{i}: 2 \leq i \leq n-1\right\}$ and $V_{2}=\left\{v_{1}, v_{n}\right\}$. Now in order to obtain $D S\left(P_{n}\right)$ from $G$, we add $w_{1}, w_{2}$ corresponding to $V_{1}, V_{2}$. Then

$$
\begin{aligned}
& \left|V\left(D S\left(P_{n}\right)\right)\right|=n+2 \text { and } \\
& E\left(D S\left(P_{n}\right)\right)=E\left(P_{n}\right) \cup\left\{v_{1} w_{2}, v_{2} w_{2}\right\} \\
& \cup\left\{w_{1} v_{i}: 2 \leq i \leq n-1\right\} \\
& \text { so } \mid E\left(D S\left(P_{n}\right) \mid=2 n-1 .\right.
\end{aligned}
$$

We define vertex labeling $f: V\left(D S\left(P_{n}\right)\right) \rightarrow\{1,2, \ldots, n+2\}$ as follows.

$$
\begin{aligned}
& f\left(w_{1}\right)=1, \\
& f\left(w_{2}\right)=4, \\
& f\left(v_{1}\right)=2, \\
& f\left(v_{2}\right)=3, \\
& f\left(v_{2+i}\right)=4+i ; \quad 1 \leq i \leq n-2
\end{aligned}
$$

In view of the above defined labeling pattern, if $n+2 \equiv 0(\bmod 16)$ then $e_{f}(0)=n-1, e_{f}(1)=n$, otherwise $e_{f}(0)=n, e_{f}(1)=n-1$.

Thus, $\left|e_{f}(0)-e_{f}(1)\right| \leq 1$.

Hence $D S\left(P_{n}\right)$ is a square divisor cordial graph.

Illustration 2.16: Square divisor cordial labeling of the graph $D S\left(P_{7}\right)$ is shown in Fig. 8.

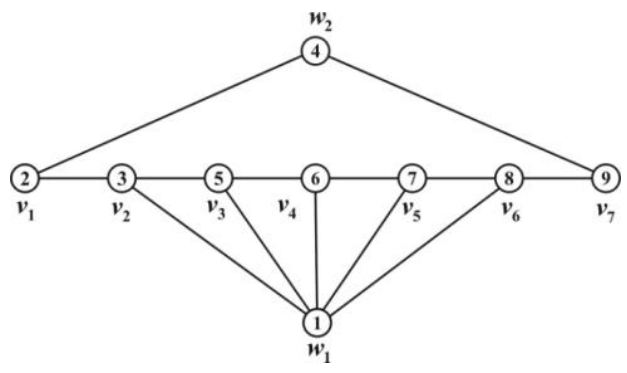

Fig. 8

\section{Concluding Remarks}

The square divisor cordial labeling is a labeling with the blend of cordial and divisor cordial labelings. As all the graphs do not admit square divisor cordial labeling, it is very interesting to find out graph or graph families which are square divisor cordial graphs. Here we contribute some new results and many graphs are proved to be square divisor cordial graphs. 


\section{References}

1. J. Gross and J. Yellen, Graph Theory and its applications (CRC Press, 1999).

2. D. M. Burton, Elementary Number Theory (Brown Publishers, $2^{\text {nd }}$ ed. 1990).

3. L. W. Beineke and S. M. Hegde, Discuss. Math. Graph Theory 21, 63 (2001).

4. V. Yegnanaryanan and P. Vaidhyanathan, J. Math. Comput. Sci. 2, 5 (2012).

5. J. A. Gallian, The Electronic J. Combin. 16 (2013), \# DS6.

6. I. Cahit, Ars Combinatoria 23, 210 (1987).

7. M. Sundaram, R. Ponraj and S. Somasundram, J. Indian Acad. Math. 27, 2 (2005).

8. S. K. Vaidya and P. L. Vihol, Modern Appl. Sci.4, 8 (2010). http://dx.doi.org/10.5539/mas.v4n8p119

9. S. K. Vaidya and P. L. Vihol, Int. J. Open Problems Computer Sci. Math. 3, 5 (2010). Available online: http://www.ijopcm.org

10. S. K. Vaidya and N. H. Shah, J. of Math. Res. 3, 4 (2011). Available online: http://www.ccsenet.org

11. S. K. Vaidya and N. H. Shah, Open J. Discrete Math. 2, 11 (2012). http://dx.doi.org/10.4236/ojdm.2012.21003

12. R. Varatharajan, S. Navanaeethakrishnan and K. Nagarajan, Int. J. Math. Combin. 4, (2011).

13. R. Varatharajan, S. Navanaeethakrishnan and K. Nagarajan, Int. Math. Forum 7, 35 (2012).

14. S. K. Vaidya and N. H. Shah, Annals Pure Appl.Math. 3, 1 (2013). Available online: www.researchmathsci.org

15. S. K. Vaidya and N. H. Shah, Annals Pure Appl.Math. 4, 2 (2013). Available online: www.researchmathsci.org

16. S. Murugesan, D. Jayaraman and J. Shiama, Int. J.Computer Appl. 64, 22 (2013).

17. P. Selvaraju, P. Balaganesan, J. Renuka and V. Balaj, Int. J. Math. Combin. 2, (2012). 